\title{
Seismic hazard analysis of Tianjin area based on strong ground motion prediction*
}

\author{
Zhao Boming ${ }^{\star}$ \\ School of Civil Engineering, Beijing Jiaotong University, Beijing 100044, China
}

\begin{abstract}
Taking Tianjin as an example, this paper proposed a methodology and process for evaluating near-fault strong ground motions from future earthquakes to mitigate earthquake damage for the metropolitan area and important engineering structures. The result of strong ground motion was predicted for Tianjin main faults by the hybrid method which mainly consists of 3D finite difference method and stochastic Green's function. Simulation is performed for 3D structures of Tianjin region and characterized asperity models. The characterized asperity model describing source heterogeneity is introduced following the fault information from the project of Tianjin Active Faults and Seismic Hazard Assessment. We simulated the worst case that two earthquakes separately occur. The results indicate that the fault position, rupture process and the sedimentary deposits of the basin significantly affect amplification of the simulated ground motion. Our results also demonstrate the possibility of practical simulating wave propagation including basin induced surface waves in broad frequency-band, for seismic hazard analysis near the fault from future earthquakes in urbanized areas.
\end{abstract}

Key words: 3D finite difference; stochastic Green's function; active fault; strong ground motion; Tianjin area CLC number: P315.9 Document code: A

\section{Introduction}

Seismic engineering evaluation based on active faults of potential earthquake relies on reasonable and accurate $3 \mathrm{D}$ prediction models such as the characterized source model and three-dimensional underground velocity structure model, with which strong ground motions can be calculated on the basis of three-dimensional calculation theory. For the calculation of high-frequency seismic motions can not be solved, analytical methods based on statistics and probability theories are employed to scientifically quantify input ground motions, analyze the exact structural and environmental dynamics, and thereby effectively reduce the earthquake disaster. Since the 1995 Kobe earthquake happened in Japan, 3D real-scale model has been introduced to simulate seismic waves, and great progress has been made in the studies on establishing rational systems (Irikura, 1986; Kamae and Irikura, 1998; Pitarka, 1999; Zhang and Chen, 2006; Zhang et al, 2008). The author put forward and im-

\footnotetext{
* Received 23 September 2009; accepted in revised form 13 June 2010; published 10 August 2010.

* Corresponding author. e-mail: bomingzhaocn@yahoo.com.cn

(C) The Seismological Society of China and Springer-Verlag Berlin Heidelberg 2010
}

proved the corresponding modeling and calculation method (Kagawa et al, 2004; Zhao et al, 2007). At present, there is no precedent in China about the application of the prediction calculation combined with active faults detection. This paper specifically gives the methodology and process combined with the calculated results from the case in Tianjin. Tianjin is a big city with key national construction, so it is significant to carry out earthquake hazard evaluation of specific faults.

\section{Prediction method}

Observation and study of amounts of strong motions can be used to calculate low-frequency ground motion based on certain source models and underground structure models, but the higher-frequency ground motions ( $>1 \mathrm{~Hz}$ generally) affected by the complex rupture of source and small-scale medium structure are characterized by randomness. Therefore, source models and true underground medium models based on deterministic method are preferred; meanwhile, numerical simulation methods such as finite element or finite difference numerical methods are developed to simulate the process of long-period seismic wave propagation so as to obtain synthesized high-frequency ground motion based on random vibration theory. The $3 \mathrm{D}$ finite difference 
method (FDM) is suitable for computing long-period ground motions instead of short-period ones. Though the empirical Green's function method or statistical Green's function method can be used to synthesize the broad frequency-band ground motion in principle, they are not expedient to characterize three-dimensional effect and long-period seismic motions with significant radiation azimuth. In this study, we synthesize the two methods and call it as hybrid method. The paper will briefly introduce the seismic ground motion evaluation system of the hybrid method.

The 3D difference method directly discretes the partial differential equation for describing motion in space-time by unit cells in study area. A generalized moment-tensor source for formulation is used, which uses a distribution of body-forces that are added to the individual components of velocity. The moment-tensor components can be represented as an equivalent distribution of body-force couples centered at the grid location. During the process of simulation, the effect of rupture propagation on the fault can also be obtained by progressive rupture of grids, then the process from rupturing of the site can be simulated. The mutually the method of staggered-grid finite difference is used (Graves, 1996).

When fault source are taken into account, compulsory external force will be exerted on the grids according to fault rupturing process. Here, asperity model is introduced to express the fault source. Because the entire object domain is embedded into the grid for modeling, it sometimes may become a high degree model according to different relations between the source and the locations. Therefore, an extremely large capacity high-speed computer and a very long CPU time are needed. To solve this kind of problem, we adopt the grid size varying (non-uniform spacing) with the model and develop the related programs, and in consequence realize the higher-than-standard speed calculation by optimizing the calculation methods. Here the A1 absorption boundary condition of (Clayton and Engquist, 1977) is used as applied to the velocity components of the wave field.

.The statistical Green's function method, which is based on analog calculation of records from middle and small-size earthquakes, is mainly applied to the regions without earthquake records. On the basis of the stochastic seismic motion synthesis (Boore, 1983) and the concept of the stochastic Green's function method (Irikura, 1986; Miyake et al, 2003), this method takes artificial waves instead of observation waves to synthesize large earthquakes. The theoretic radiation characteristics of seismic waves tend to be passivated in high frequency domain, because of dispersion and reflection of waves, resulting in the nearly isotropic radiation pattern. Therefore, in order to effectively reflect this kind of radiation pattern (phenomenon) in the analog calculations, the frequency-dependent source rupture radiation characteristic model is used to improve elementary seismic methods.

Fourier spectrum of ground acceleration is adopted to express the source spectrum (Boore, 1983). Based on the white noise time series produced by the chaotic number, a certain time window function, whose Fourier spectrum takes a shape of the $\omega^{-2}$ law, is implemented to the white noise. The spectrum adjusts the amplitude of vibration according to the value of seismic moment, and by inverse Fourier transformation, we can get synthetic waveforms that satisfy the $\omega^{-2}$ law. Disseminating the scenario source waveform to the bedrock under the engineering site, we can obtain the source waveform of the bedrock at the site.

Although the above equation only expresses the change in the amplitude of vibration, we can obtain the artificial seismic wave under the site which is mixed with the effect of propagation path by adjusting the phase of waveform with delayed propagation time. Finally, according to the stochastic Green's function method (Irikura, 1986), we can synthesize ground motions for large earthquakes. Regarding the sites, we can compute the surface seismic waves basing the underground structure model by the same method.

Long-period seismic waves obtained by $3 \mathrm{D}$ finite difference method and short-period seismic waves computed by random finite fault model are cross-filtered between frequency bands short-period and long-period (generally $0.5-1.0 \mathrm{~s}$ ), separately, then they are superimposed in frequency domain, and transformed into the wide-frequency-band ground motion time history by Fourier transformation.

\section{Source and structural models}

According to the results by the project of Tianjin Active Faults and Seismic Hazard Assessment (Zhao, 2008, Zhou, 2008), the target faults are determined as the north segment of Cangdong fault $\left(F_{1}\right)$ and the south Tianjin fault $\left(F_{2}\right)$ with an estimated magnitude of 6.4 and 6.1 on the Richter scale (see Figure 1). According to the exploration results of active faults, we make the 
characterized asperity model describing source heterogeneity for strong ground motions of the designated faults (Somerville et al, 1999; Miyake et al, 2003), and set up different seismotectonic models and the spatial locations (seismogenic zones) considering the uncertainty of the findings and the source model. The dip angle of $F_{1}$ is $65^{\circ}-70^{\circ}$, and the slip is about $210^{\circ}\left(30^{\circ}\right)$. The other faults represent the different source schemes. The above fault scheme fully considers the negative impact on the urban areas. The different source locations are determined to illustrate the uncertainty of the fault locations according to the results of fault activity survey (Zhao, 2008).

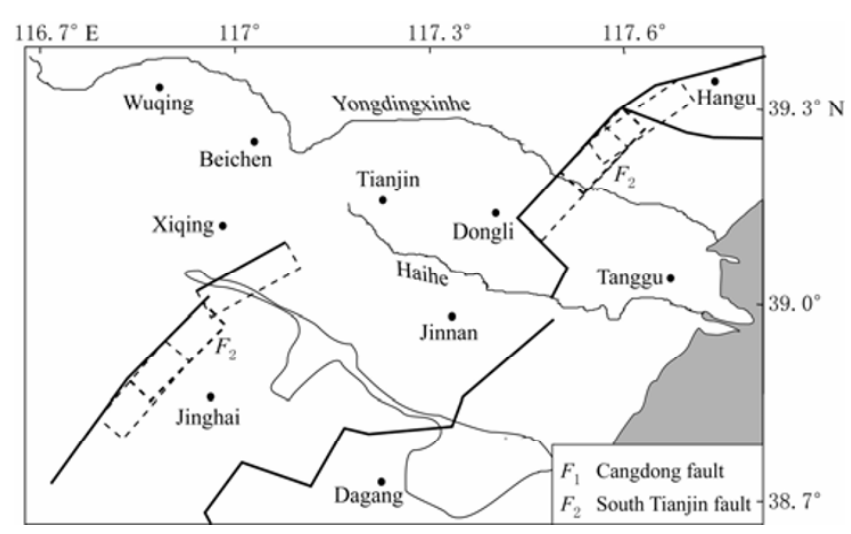

Figure 1 Location of objective faults in Tianjin region.

The Tianjin 3D underground structure model covers an area of roughly $7800 \mathrm{~km}^{2}$ (about $80 \mathrm{~km}$ from the south to the north, $97.5 \mathrm{~km}$ from the east to the west, and $70 \mathrm{~km}$ in depth, with geographic coordinates $38^{\circ} 40^{\prime} \mathrm{N}-39^{\circ} 23^{\prime} \mathrm{N}$ and $\left.116^{\circ} 41^{\prime} \mathrm{E}-117^{\circ} 50^{\prime} \mathrm{E}\right)$. The layered Tianjin structure model is mainly composed of Quaternary stratum, Neogene stratum and the crust speed structural model. Referring to results about exploration and drilling, the standard stratum is divided into $\mathrm{Q}_{8}, \mathrm{Q}_{5}$, $\mathrm{Q}_{3}, \mathrm{Q}_{1}$ and $\mathrm{Q}$ stratum which is the basement of Quaternary. The bottom layers of $Q$ layer, that is, $T_{1}, T_{2}, N_{1}$ and seismogenic zone, are potential for earthquake occurrence. The underground speed structural model is of stratification, and each floor is characterized by seven parameters, including space coordinates $\left(x_{j}, y_{j}, z_{j}\right)$ of medium factor. The quality factor $Q_{\mathrm{PS}}$ is empirically determined. The parameters of crust structure are set according to geological data, as shown in Table 1.

The detailed conditions of source model and underground structural model refer to the related study reports (Zhao, 2008; Zhou, 2008).
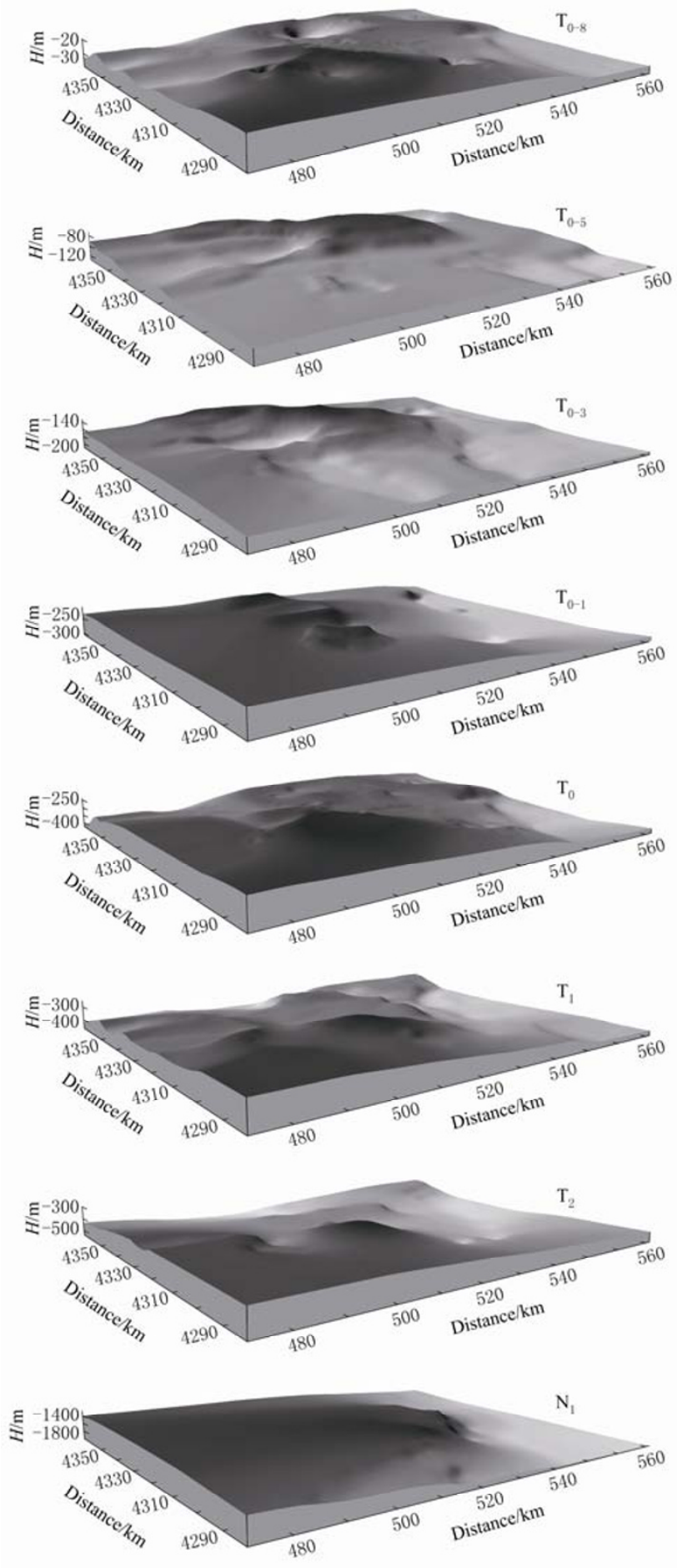

Figure 2 Strata spatial connection of underground velocity structure model of Tianjin. $H$ denotes the elevation.

\section{Result analyses}

Assuming the $\mathrm{S}$ wave velocity of surface layer of the Tianjin area is $200 \mathrm{~m} / \mathrm{s}$, the thickness of 
Table 1 Physics parameter of underground velocity structuremodel

\begin{tabular}{ccccc}
\hline Layer & $v_{\mathrm{P}} /\left(\mathrm{km} \cdot \mathrm{s}^{-1}\right)$ & $v_{\mathrm{S}} /\left(\mathrm{km} \cdot \mathrm{s}^{-1}\right)$ & $\rho /\left(\mathrm{g} \cdot \mathrm{cm}^{-3}\right)$ & $Q_{\mathrm{PS}}$ \\
\hline $\mathrm{T}_{0-8}$ & $1.00-1.20$ & $0.20-0.30$ & $1.70-1.75$ & $50-60$ \\
$\mathrm{~T}_{0-5}$ & 1.30 & 0.40 & 1.80 & 80 \\
$\mathrm{~T}_{0-3}$ & 1.70 & 0.56 & 1.85 & 100 \\
$\mathrm{~T}_{0-1}$ & 2.10 & 0.75 & 2.00 & 150 \\
$\mathrm{~T}_{0}$ & 2.50 & 1.00 & 2.10 & 300 \\
$\mathrm{~T}_{1}$ & 3.60 & 1.60 & 2.20 & 500 \\
$\mathrm{~T}_{2}$ & 4.40 & 2.20 & 2.40 & 500 \\
$\mathrm{~N}_{1}$ & 5.10 & 2.80 & 2.60 & 500 \\
Seismogenic & $5.40-6.00$ & $3.00-3.20$ & $2.80-2.90$ & 1000 \\
zone & & & & \\
\hline
\end{tabular}

overburden layer varies complicatedly between $300 \mathrm{~m}$ and $1000 \mathrm{~m}$, the area is the typical alluvial soft soil structure. To ensure the effective computation period, the area is gridded as densely as possible to objectively reflect the accurate shape of the underground model. The computed model is $100 \mathrm{~km}$ in EW, $85 \mathrm{~km}$ in NS, and $50 \mathrm{~km}$ in depth. The grid cell in horizontal direction is set to be $50 \mathrm{~m}$ and that in vertical direction adopts variable spacing. In the scope of settled layer depth, the grid size is $10-50 \mathrm{~m}$, and $100-200 \mathrm{~m}$ in the bedrock. The model consists of $9.0 \times 10^{7}$ grid points. Because of the densely-spaced grid division of the surface layer of the model, it requires a short step-interval for the computation to satisfy the required computational accuracy. When the largest $\mathrm{P}$ wave velocity is $v_{\mathrm{P}}=6 \mathrm{~km} / \mathrm{s}$, the surface layer grid size is $20 \mathrm{~m}$, the time step requested is not longer than $0.002 \mathrm{~s}$. The period ranges $1.0-20.0 \mathrm{~s}(1.0-0.05 \mathrm{~Hz})$. The long-period seismic wave is calculated by staggered-grid finite difference with variable spacing, and the calculation of short-period seismic wave is based on the improved stochastic Green's function method. Then the seismic wave of short-period is synthesized as wideband seismic wave by filtering and superimposing in the scope of frequency. During the computing process, the computed velocity of grid node on the model surface is output, which facilitates the intuitionistic analysis of spatial propagation and distribution of seismic motion through visualized observation of spatial fluctuations of seismic waves.

By comprehensive analysis of the propagation process and spatial distribution of ground motions, it can be found that wave surface is curved some times during propagation process. The variation of wavelength in the wavefield indicates that underground structure has a remarkable influence on ground motions. The first rea- son for it is the weak relation among stratums due to the stratums with great up-and-down and the great difference in shapes in the region; the second reason is that there are more independent subsidence centers in the region. The complex underground structure leads to great changes of ground motion space.

The seismic waves all have a longer durable time with the longest time over $10 \mathrm{~s}$. At the same time, the abundant surface wave in the waveform reflects the geological surface characteristic of thicker soft overlay in Tianjin region. The seismic waveform shows that in the source region in the southwest, the waves have the larger amplitude in front of fault rupture, and take on a weaken tendency along with the source distance. It also shows that the waveform has the characteristics of short period and small amplitude in the rear of fault rupture, which reflects the source effect and characteristics of fault rupture.

Figure 3 shows distribution of PGA in the calculation range when the north segment of Cangdong fault $F_{1}$ with a magnitude of 6.4 occurs. From Figure 3, it can be seen that the high-value influence region is extensively distributed. The main high-value influence region is around the fault. The extensive distribution along the northeast expresses the rupture effect of dextrorotary strike-slip fault along the fault. As a result of the larger fault inclination, the normal fault effect is relatively weak. The limit of the maximum acceleration amounts to about $3.5 \mathrm{~m} / \mathrm{s}^{2}$ in the source region. As the fault margin is about $10 \mathrm{~km}$ away from the Tianjin urban area, the earthquake has some influence on the central urban area within the Outer Ring of Tianjin municipality, and the maximum acceleration is $0.2-1.2 \mathrm{~m} / \mathrm{s}^{2}$. The faults with potential earthquake are near Hangu, so the earthquake has great influence on Hangu region and the western regions. The earthquake also has some influence on Tanggu region where the acceleration is about $0.2-1.0$ $\mathrm{m} / \mathrm{s}^{2}$. The maximum value amounts to $1 \mathrm{~m} / \mathrm{s}^{2}$ outside the Tanggu, but the effect is relatively small in the costal areas.

The PGV and PGD (peak ground displacement) show that the distribution scope of high-value influence region is larger. The main high-value influence region is around the fault. The high-value distribution mechanism is the same as the above analysis of the PGA basically. From the distribution of the maximum value, the region where high-value influence area exists has a close relation with the mechanism of loose overlay and fault. 


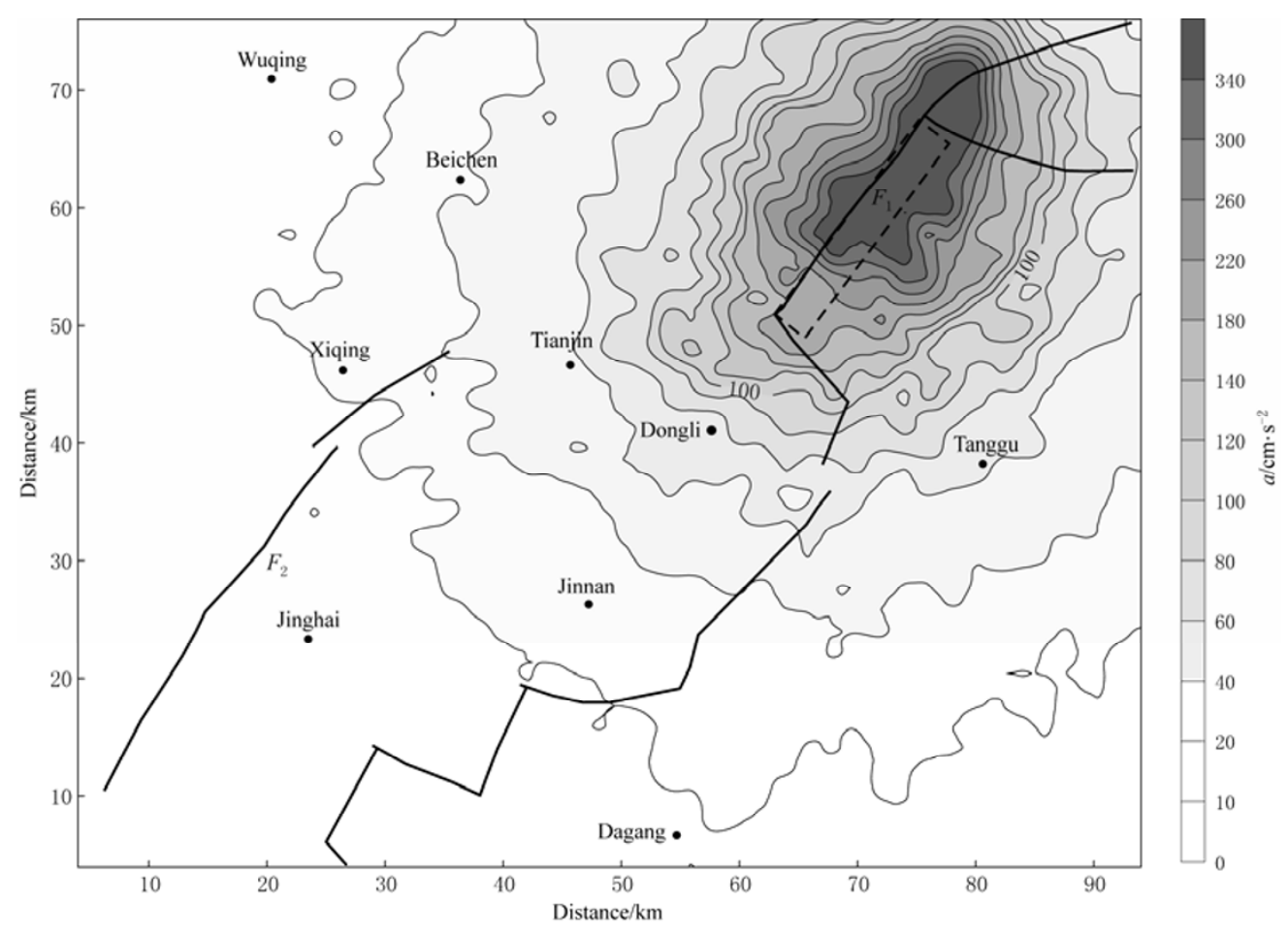

Figure 3 PGA map of Cangdong fault $F_{1}$.

Figure 4 shows PGA distribution of the south Tianjin fault $F_{2}$ (M6.1). The source mechanism of the fault $F_{2}$ is the same as that of the Cangdong rupture, but the difference in magnitude and underground structures is great, so the distributions of ground motions between them are obviously different. The north segment of Cangdong rupture $F_{1}$ is $22 \mathrm{~km}$ in length. Because the upper structure is located in the stratums tilted to seaside, and the underground structure is more complex than the south Tianjin rupture region, the distribution characteristics and influences of ground motions are different so that they need to be treated differently. The ground motions near the field, especially near the down-type faults, have very complicated characteristics. The surface waves are abundant in the waveform components of Tianjin target areas, at the same time, the long durable time and abundant medium-and-long period waves are existent. Different durable times and frequency spectra will have different destruction effect on buildings.

After calculating the PGA, PGV and PGD of seismic ground motions, the ground motion response (intensity) regionalization of the scenario earthquakes is done by taking 1 square kilometer as zonation unit in order to provide convenience for engineering application. ID value is assigned to the zonation unit according to the spatial position. The zonation unit indicates the mean maximum acceleration, history acceleration time and response spectrum (acceleration, velocity, displacement), and is called the potential earthquake hazard map. Maximum velocity and displacement distribution based on regionalization can be done according to the specific use. Figure 5 shows the distribution of potential seismic hazard based on the prediction results for the north segment of Cangdong fault.

\section{Discussion and conclusions}

The paper presented process of seismic hazard analysis based on the prediction for near-fault strong ground motions from future earthquakes in urbanized areas. On the background of Tianjin which is the key defensive area in China, wideband strong ground motion field of the north segment of Cangdong fault (M6.4) and the south Tianjin rupture (M6.1) is predicted with the help of the hybrid method mainly consisting of 3D finite difference method and the improved stochastic Green's function method. The prediction method is based on 3D underground structural model of Tianjin and characterized source model according to the project of Tianjin Active Fault Survey and Hazard Evaluation. The prediction results indicate that wave field of strong ground 


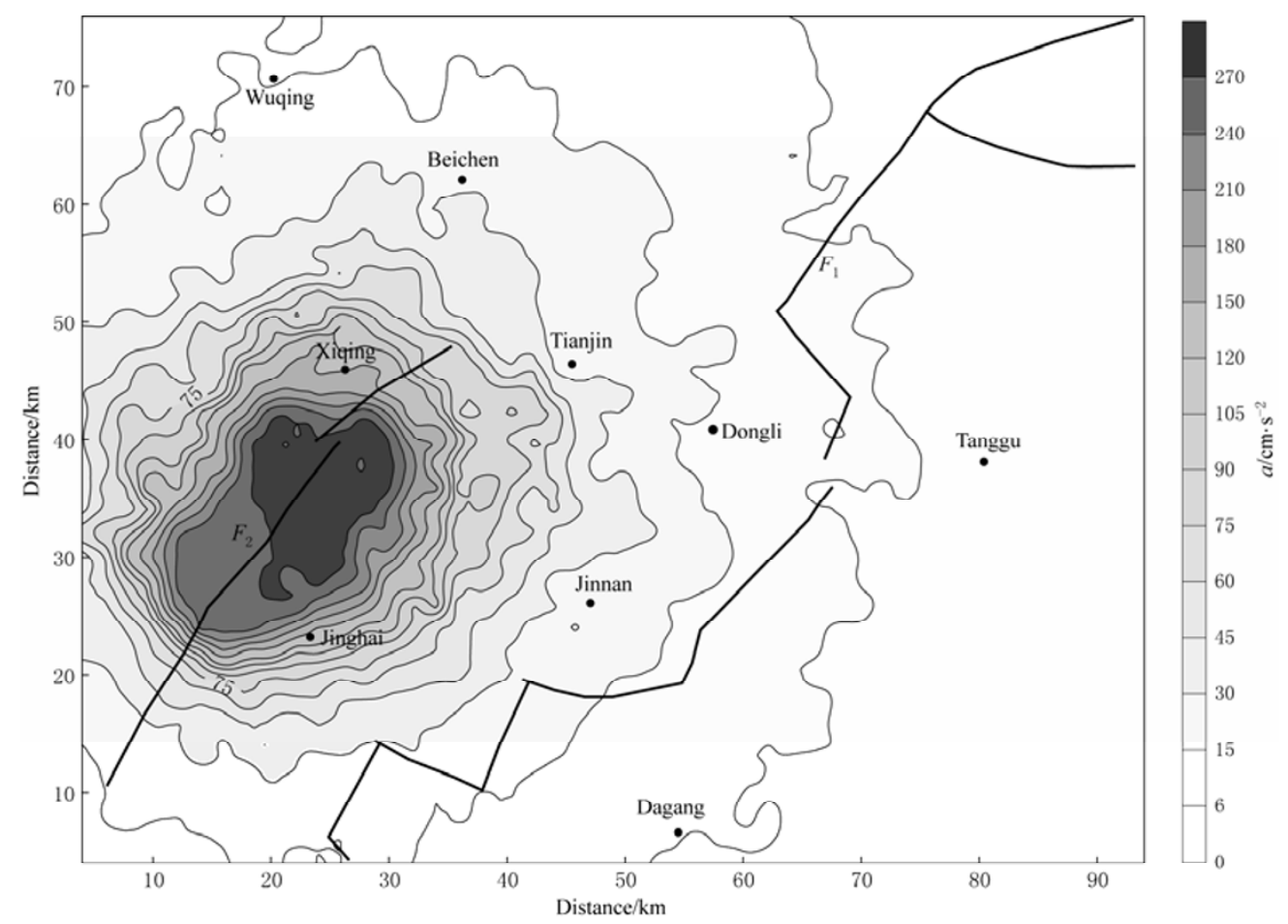

Figure 4 PGA map of south Tianjin fault $F_{2}$.

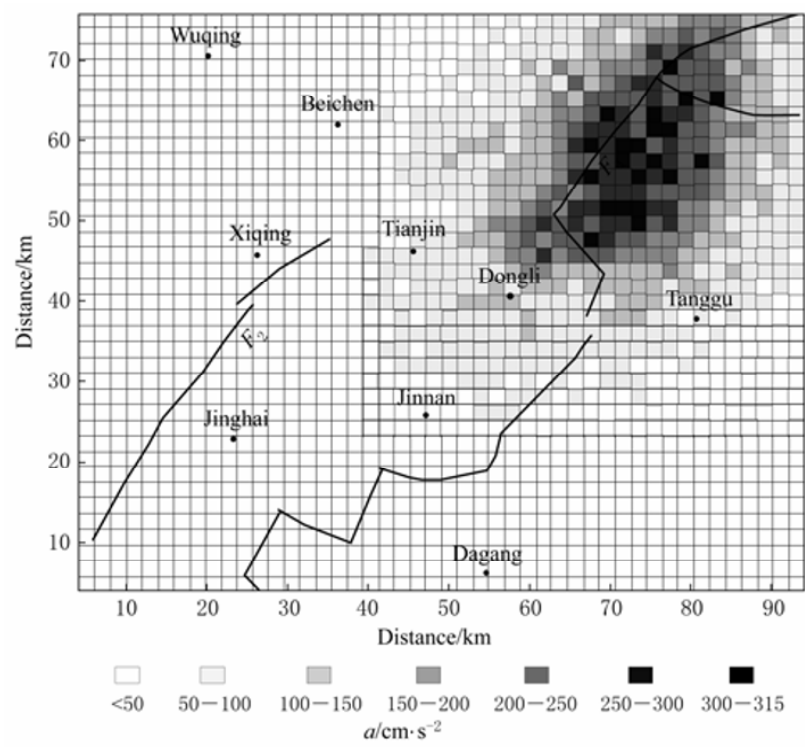

Figure 5 Seismic hazard map based on prediction result for Cangdong $F_{1}$ fault.

motions have remarkable influence on underground structure and seismogenic structure. This method reflects the nature of strong ground motion field for wideband including the complex surface waves produced by soft soil overburden and provides the possibility of metropolitan seismic hazard evaluation based on strong ground motion field near the fault considering regional environmental conditions. The method also provides scientific basis for long and short term plan of metropolitans based on the earthquake hazard evaluation according to ground motions intensity regionalization on specific cell by predicting the strong ground motion.

The proposed prediction method of strong ground motions considers the source characteristics, propagation route characteristics and field amplification characteristics. It contains the other relevant characteristics that should be considered in strong ground motions prediction. It also considers the characteristics of city down-type earthquakes and ground motions near the field, and the influences of 3D structure and site effect and so on. Based on the source mechanism, and on the condition of setting the potential maximum magnitude of source faults identified by active faults detection, detailed ground motion prediction and analysis are carried out under the current possible scientific level.

The present standard cannot reflect the complex change of seismic waves, and this kind of uncertainties will bring challenge to the earthquake resistance of civil engineering. Therefore, it is of importance to understand and correctly evaluate the characteristics of earthquake ground motion, and to carry on the reasonable dynamics analysis and seismic design. This paper assumes earth- 
quake as the low probability event, and adopts greatest magnitude of assumed goal fault.

Acknowledgments This study is supported by the National Natural Science Foundation of China (40674016), Earthquake Research Fund of Ministry of Science and Technology of China ((2007)203/8-53).

\section{References}

Boore D (1983). Stochastic simulation of high-frequency ground motions based on seismological models of the radiated spectra. Bull Seism Soc Amer 73: 1 865-1 894.

Clayton R and Engquist B (1977). Absorbing boundary conditions for acoustic and elastic wave equations. Bull Seism Soc Amer 67: 1 529-1 540.

Graves R (1996). Simulating seismic wave propagation in 3D elastic media using staggered-grid finite differences. Bull Seism Soc Amer 86: 1 0911106.

Hanks T C and Kanamori H (1979). A moment magnitude scale. J Geophys Res 84: 2 348-2 350.

Irikura K (1986). Prediction of strong acceleration motion using empirical Green's function. In: Proc of the 7th Japan Earthq Eng Symp. Steering Committee of the 7th Japan Earthquake Engineering Symposium, Tokyo, $151-156$.

Kagawa T, Zhao B M, Miyakoshi K and Irikura K (2004). Modeling of 3D basin structures for seismic wave simulations based on available information on the target area: Case study of the Osaka Basin, Japan. Bull Seism Soc Amer 94: $1353-1368$.
Kamae K and Irikura K (1998). Source model of the 1995 Hyogo-ken Nanbu earthquake and simulation of near-source ground motion. Bull Seism Soc Amer 88: 400-412.

Miyake H, Iwata T and Irikura K (2003). Source characterization for broadband ground-motion simulation: Kinematic heterogeneous source model and strong motion generation area. Bull Seism Soc Amer 93: 2 531-2 545.

Pitarka A (1999). 3D elastic finite-difference modeling of seismic motion using staggered grids with nonuniform spacing. Bull Seism Soc Amer 89: 54-68.

Somerville P G, Irikura K, Graves R, Sawada S, Wald D, Abrahamson N, Iwasaki Y, Kagawa T, Smith N and Kowada A. (1999). Characterizing crustal earthquake slip models for the prediction of strong ground motion. Seism Res Lett 70: $59-80$.

Zhang W and Chen X F (2006). Traction image method for irregular free surface boundaries in finte difference seismic wave simulation. Geophys J Int 67: 337-353.

Zhang W, Shen Y and Chen X F (2008). Numerical simulation of strong ground motion for the $M_{\mathrm{S}} 8.0$ Wenchuan earthquake of 12 May 2008. Science in China (Series D) 51(12): 1 673-1 682.

Zhao B M (2008). Report on the Seismic Hazard Assessment for Main Active Faults in Tianjin Area. Report of Beijing Jiaotong University, Beijing, 1-150 (in Chinese).

Zhao B M, Kagawa T and Tsurugi M (2007). Study on long-period strong motion simulation for large-scale earthquakes. J Geophys Eng 4(3): 301-307, doi:10.1088/1742-2132/4 /3 S09.

Zhou Q (2008). Report on the Risk Assessment for Main Active Faults in Tianjin Area. Report of Institute of Geology of China Earthquake Administration, Beijing, 1-122 (in Chinese). 